\title{
Eficiencia en el uso de la radiación en papa estimada a partir de la cobertura del follaje
}

\author{
de la Casa, A.; G. Ovando, L. Bressanini, J. Martínez y A. Rodríguez
}

\begin{abstract}
RESUMEN
La eficiencia en el uso de la radiación (EUR) de un cultivo es la relación entre la materia seca producida y la radiación fotosintéticamente activa interceptada (RFAl) durante su ciclo. La fracción de radiación fotosintéticamente activa interceptada (fRFAl) puede determinarse mediante el método tradicional de Beer, a partir del índice de área foliar (iaf), o empleando la cobertura del suelo ( $f$, como medida subrogante de fRFAl. Cuando el iaf en papa supera 3, el valor de fRFAI cambia muy poco, haciendo muy difícil detectar diferencias debidas a variaciones en las condiciones del cultivo. El objetivo de este trabajo fue determinar la EUR en papa (Solanum tuberosum L.) cv. Spunta, comparando el uso de valores de iafy de fpara obtener fRFAI. El ensayo se realizó en el cinturón verde de Córdoba, Argentina, sobre un cultivo de ciclo tardío entre febrero y mayo de 2008. La utilización del valor de $f$ produjo resultados que sobreestiman EUR, como consecuencia de una sistemática subestimación de fRFAl, mientras que tomando valores de $f R F A /$ corregidos de acuerdo a la relación con $f$ previamente establecida, EUR resultó similar a la obtenida con el método de referencia, que presentó un valor de 2,90 $\mathrm{gr} \mathrm{MJ}^{-1}$ PAR.
\end{abstract}

Palabras clave: papa, cobertura de follaje, índice de área foliar, EUR.

de la Casa, A.; G. Ovando, L. Bressanini, J. Martínez and A. Rodríguez, 2011. Radiation use efficiency in potato crop estimated from the foliage coverage. Agriscientia XXVIII: 21-30

\section{SUMMARY}

The radiation use efficiency ( $R U E$ ) of a crop is the relationship between dry matter produced and the intercepted photosynthetically active radiation (IPAR) during the growth cycle. The IPAR can be determined by applying Beer's traditional method, that uses leaf area index $(L A l)$, or taking ground cover $(f)$ as a surrogate of IPAR. When potato $L A /$ exceeds 3 , the IPAR values change very little, making it very difficult to detect differences due to variations in crop conditions. The aim of this study was to determine the RUE in potato (Solanum tuberosum L.) cv. Spunta, comparing the use of $L A l$ and $f$ values to obtain IPAR. The trial was con- 
ducted in the green belt of Cordoba, Argentina, on a late season crop from February to May 2008. The use of $f$ values overestimates $R U E$, as a result of a systematic underestimation of IPAR, but when IPAR values where correctly taken according to the previously established relationship with $f, R U E$ was similar to that obtained through the reference method which presented a value of $2.90 \mathrm{~g} \mathrm{MJ}^{-1}$ PAR

Key words: potato, foliage cover, leaf area index, RUE

A. de la Casa, A. G. Ovando, L. Bressanini, J. Martínez y A. Rodríguez. Universidad Nacional de Córdoba, Facultad de Ciencias Agropecuarias. CC 509, 5000 Córdoba, Argentina. Correspondencia a: delacasa@agro.unc.edu.ar

\section{INTRODUCCIÓN}

La cantidad de radiación solar absorbida por la vegetación y la utilización de esta energía en el crecimiento vegetal constituyen los procesos biofísicos que controlan la producción de biomasa y el rendimiento de los cultivos a una tasa potencial. Por esta razón, estos parámetros son fundamentales para estimar la cantidad de biomasa acumulada y monitorear el crecimiento de un cultivo (Kooman et al., 1996; Liu et al., 2004), en la medida que no experimente condiciones de estrés durante su ciclo (Monteith, 1977; Sinclair \& Muchow, 1999).

La pendiente de la relación lineal entre los valores acumulados de la materia seca vegetal y la radiación solar (total o fotosintéticamente activa), absorbida o interceptada, es denominada eficiencia en el uso de la radiación (EUR, gr $\mathrm{MJ}^{-1}$ ) (Monteith, 1977). En general, se puede asumir que el valor de radiación interceptada se asemeja a la cantidad de energía absorbida, de manera particular cuando el vegetal no presenta tejidos en senescencia (Gallo et al., 1993; Edwards et al., 2005). También es común que la radiación solar se exprese en forma total, o se restrinja sólo al espectro fotosintéticamente activo (RFA) (Sinclair \& Muchow, 1999). En un sentido aplicado, los modelos de simulación de cultivos, ya sean específicos para papa como SUBSTOR (Griffin et al., 1993) o genéricos como STICS (Brisson et al., 2003), estiman la producción diaria de materia seca a tasa potencial como el producto entre la cantidad de radiación solar y un valor particular de EUR.

La EUR es una medida que tiende a ser estable en papa (Bouman et al., 1992) y se ha mostrado invariable incluso bajo diferentes niveles de ataque de tizón tardío (Phytophthora infestans) (Van Oijen, 1991). Jefferies \& Heilbronn (1991) en su modelo de productividad utilizan un valor de $1,75 \mathrm{~g} \mathrm{MJ}^{-1}$ (solar), que corresponde a la tasa máxima para el cultivo de papa, de acuerdo a distintas experiencias reportadas por Sinclair \& Muchow (1999). Sin embargo, Kooman et al. (1996) señalan para EUR un rango entre 1,8 y 3,7 $\mathrm{g} \mathrm{MJ}^{-1}$ (RFA) en ensayos correspondientes a distintos genotipos, lugares geográficos y estaciones de cultivo. Asimismo, el modelo SUBSTOR (Griffin et al., 1993) utiliza para estimar la tasa potencial de crecimiento un valor de 3,5 $\mathrm{g} \mathrm{MJ}^{-1}$ (RFA) antes de la tuberización, que se incrementa posteriormente a $4 \mathrm{~g} \mathrm{MJ}^{-1}$ (RFA).

La naturaleza empírica de la EUR y la baja precisión con la que puede medirse son causas de incertidumbre acerca de las estimaciones que se obtienen a partir de los modelos de simulación de cultivos; en este sentido, algunos autores cuestionan la utilización del concepto de EUR en la modelación de cultivos (Loomis \& Amthor, 1999). La forma en que se deberían realizar las mediciones a los efectos de estimar EUR, tanto de la biomasa del cultivo como de la intercepción de radiación, ha sido objeto de abundante debate. Si bien Sinclair \& Muchow (1999) sostienen que la relación lineal entre la biomasa acumulada y el total de la radiación interceptada para el mismo periodo provee una expresión apropiada de EUR, y a pesar de ser el procedimiento más utilizado en su determinación, su naturaleza acumulativa ha sido objetada y su implementación puesta en tela de juicio (Demetriades-Shah et al., 1992, 1994; Monteith, 1994). Sin embargo, la EUR es un parámetro ecofisiológico particularmente útil a los efectos de analizar el crecimiento vegetal (Kadaja \& Tooming, 2004, Lindquist et al., 2005; de la Casa et al., 2007).

Entre las distintas alternativas para medir la fracción de radiación interceptada, el empleo del 
medidor de fotones fotosintéticamente activos o "interceptómetro" ha sido una de las técnicas más utilizadas (Jonckheere et al., 2004), cuyo empleo permite, además, estimar el valor del índice de área foliar (iaf) en forma no destructiva. En papa y bajo las condiciones ambientales y de producción del cinturón verde de Córdoba, Argentina, este instrumento se ha utilizado para evaluar la EUR (de la Casa et al., 2007), así como para estimar el iaf bajo diferentes densidades de plantación (de la Casa et al., 2008a).

Empleando una analogía con la ley de Beer, Monsi \& Saeki (2005) determinaron la fracción de radiación interceptada a partir del valor de iaf y de un coeficiente de extinción lumínico $(K)$, que depende del ángulo de inserción de las hojas. La relación entre el iaf y la fracción de radiación interceptada, sin embargo, presenta un efecto de saturación típico a medida que se incrementa la superposición de hojas y con ella el área foliar. En papa, los estudios muestran que cuando el iaf supera el valor de 3 la fracción de radiación interceptada cambia muy poco (Jefferies \& Heilbronn, 1991; de la Casa et al., 2007), haciendo muy difícil la detección de diferencias que se pueden suscitar entre distintas condiciones propias del manejo del cultivo, como la densidad de siembra, distancia entre plantas, distancia entre hileras.

La fracción de suelo cubierto por el follaje (f) ha sido interpretada en algunos cultivos como una medida subrogante de la fracción de radiación interceptada (Steven et al., 1986). Purcell (2000) en soja, Caviglia et al. (2003) en trigo y Edwards et al. (2005) en maíz evaluaron esta premisa que tiene la ventaja de requerir sólo fotografías digitales obtenidas de manera vertical sobre el cultivo a los efectos de determinar la condición de cobertura, y es independiente de las condiciones de iluminación. En papa, Haverkort et al. (1991) utilizando una grilla regular y de la Casa et al. (2007) por medio de fotografías digitales, han mostrado que existe una asociación estrecha entre ambas variables. Por otra parte, el modelo de cultivo STICS, entre las distintas variantes de cálculo que dispone, permite utilizar directamente el valor de $f$ para expresar fracción de radiación fotosintéticamente activa interceptada (fRFAl) y, de este modo, estimar el rendimiento del cultivo (Brisson et al., 2003).

Purcell (2000) estableció que la relación entre $f$ y $f R F A$ l en soja se aproxima a la función de identidad, en tanto Kooman et al. (1996), asumiendo un comportamiento similar para papa, determinaron la cantidad de radiación fotosintéticamente activa (RFA) interceptada por el cultivo directamente como el producto de $f$ y la RFA incidente. de la
Casa et al. (2008b) mostraron que el empleo de la cobertura de follaje para estimar fRFAl constituye una alternativa apropiada para monitorear el crecimiento del cultivo de papa bajo diferentes densidades de plantación; sin embargo, estos autores encontraron que la relación entre fRFAl y $f$ no resultó lineal, y verificaron que el coeficiente de extinción (cociente entre los coeficientes de extinción obtenidos con el interceptómetro y la cobertura $(\mathrm{ki} / \mathrm{kf})$ ) adopta un valor de 0,7

El objetivo de este trabajo fue determinar la EUR en papa comparando dos alternativas para calcular la fracción de radiación interceptada; la de referencia, aplicando el modelo de Beer mediante el uso de valores de iaf, o empleando directamente valores de $f$ corregidos por el método propuesto por de la Casa et al. (2008b).

\section{MATERIALES Y MÉTODOS}

El trabajo se realizó en un cultivo de papa (Solanum tuberosum L.) cv. Spunta, durante la campaña semitardía (de febrero a mayo) de 2008, en un lote de producción comercial del cinturón verde de la ciudad de Córdoba, Argentina (3130' lat. Sur, 6408' long. Oeste, 402 m s.n.m.). El suelo del sector se clasificó como Haplustol éntico, serie Manfredi, limosa fina, mixta, térmica (Jarsún et al., 2006), sin limitaciones para la agricultura bajo riego. El cultivo se realizó bajo riego periódico por inundación y se le aplicaron $350 \mathrm{~kg} \mathrm{ha}^{-1}$ de un fertilizante complejo $20-17-3+4 \%$ S y $1 \%$ Mg en plantación, y $100 \mathrm{~kg} \mathrm{ha}^{-1}$ de 22-0-0 +9\% S y 4,5\% Ca al inicio de floración. La experiencia se llevó a cabo con diferentes densidades de plantación, a los efectos de analizar la evolución de la cobertura del follaje ( $f$, \% o fracción), el índice de área foliar (iaf, $\mathrm{m}^{2} \mathrm{~m}^{-2}$ ) y la fracción interceptada de radiación fotosintéticamente activa (fRFAl) a lo largo del ciclo.

La plantación se realizó el 12 de febrero de 2008, en forma mecánica, con la densidad habitual promedio del productor de cinco propágulos por $\mathrm{m}^{2}$. El mismo día, en una parcela de cuatro camellones separados a 0,80 m $\times 25 \mathrm{~m}$ de longitud, se incorporaron manualmente en forma equidistante cinco propágulos más por $\mathrm{m}^{2}$, denominándolo tratamiento de doble densidad (DD). En la etapa de plena emergencia, observada 14 días posteriores a la plantación, en una parcela apareada a DD se procedió al arrancado manual de tres tallos por cada $\mathrm{m}^{2}$, constituyendo el tratamiento de baja densidad o raleado (DR). Los tratamientos DD y DR se compararon apareados a una parcela similar sin alterar o testigo con la densidad habitual promedio del productor a la que se denominó tratamiento de densidad comercial (DC). El 13 de marzo las 
tres densidades de tallos emergidos en promedio fueron: 17 tallos $\mathrm{m}^{-2}$ (DD), 11 tallos $\mathrm{m}^{-2}$ (DC) y 6 tallos $\mathrm{m}^{-2}(\mathrm{DR})$. Este esquema no se replicó para evitar alterar la modalidad de prácticas culturales del productor.

Durante el período entre inicio de emergencia y hasta final de ciclo del cultivo, cada 15 días se recolectó el total de la biomasa aérea y radicular de 1 $\mathrm{m}^{2}$ en cada parcela experimental para determinar la materia seca acumulada de cada tratamiento.

Las condiciones de temperatura y humedad relativa del aire a $0,40,0,80$ y $1,2 \mathrm{~m}$ de altura y de temperatura del suelo a 0,05, 0,10 y 0,20 m de profundidad, se monitorearon durante todo el experimento con una estación automática instalada en la parcela del ensayo. La estación también registra la humedad del suelo a partir de tres sensores de matriz granular dispuestos a 0,10, 0,20 y 0,30 m de profundidad. Los registros diarios de humedad de suelo en contadores digitales (CD) se expresaron en forma volumétrica (HS), utilizando una curva de calibración obtenida previamente para estas condiciones (Rodríguez et al., 1997). Considerando un perfil de 0,50 $\mathrm{m}$ de profundidad y un valor de capacidad de campo (CC) de 28\%, típico para este suelo (Dardanelli et al., 1997), la deficiencia de agua se calculó de acuerdo a CC-HS.

La reducción de la EUR como consecuencia del estrés hídrico en papa se calculó sobre la base de la función que describen Jefferies \& Mackerron (1989).

Los datos de radiación solar total recibida (RSr) proceden de una estación automática distante a 9 $\mathrm{km}$ del área experimental y se presentan acumulados diariamente. La RS astronómica (RSa) se calculó de acuerdo a las ecuaciones de Duffie \& Beckman (1980). De la radiación solar total recibida, se consideró que solo el 50\% corresponde a la fracción de radiación fotosintéticamente activa (Sinclair \& Muchow, 1999), si bien este valor puede cambiar de acuerdo a la cobertura de nubes, presencia de bruma y hora del día.

Las determinaciones de fRFAl se realizaron a partir de un contador del flujo de fotones fotosintéticamente activos o "interceptómetro" (PAR/LAI Ceptometer, Decagon Devices, Pullman, Washington, USA), efectuando en cada fecha seis repeticiones por tratamiento (con la barra dispuesta de manera perpendicular al surco), en distintos sectores de cada parcela experimental. Los valores de fRFA/ se obtuvieron con la siguiente expresión:

$$
f R F A I=1-\left(\frac{F R F A_{\text {abajo }}}{F R F A_{\text {arriba }}}\right)
$$

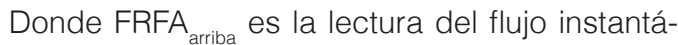
neo de radiación fotosintéticamente activa medida

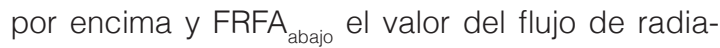
ción de un promedio de tres lecturas realizadas por debajo del dosel. Las observaciones se realizaron durante el ciclo con una frecuencia promedio de 12 días, sólo bajo condiciones de cielo despejado y durante las horas próximas al mediodía solar, lo que permitió homogeneizar las mediciones (de la Casa et al., 2007).

Los valores de iaf se calcularon a partir de las lecturas del "interceptómetro", por medio de la siguiente expresión (de la Casa et al., 2007):

$$
\text { iaf }=\frac{\left[\left(1-\frac{1}{2 K}\right) f b-1\right] \operatorname{Ln}\left(\frac{F R F A_{\text {abajo }}}{F R F A_{\text {arriba }}}\right)}{A(1-0,47 \mathrm{fb})}
$$

de $\mathrm{K}$ es el coeficiente de extinción lumínico que, al considerar un parámetro de distribución angular esférico, simplifica su cálculo a $K=1 /\left(2^{*} \cos v\right)$, siendo $v$ el ángulo cenital del lugar, día y hora de la medición; fb es la fracción de la radiación directa con respecto a la radiación solar total recibida, que se consideró igual a 0,9 (de la Casa et al., 2008a) por ser mediciones realizadas en días completamente despejados; y A es un coeficiente de absorción general del dosel que resulta igual a 0,86 para un valor de absortividad de 0,9 (AccuPAR, 2001).

A partir de los valores estimados de iaf, la fRFAl_iaf se calculó con la fórmula clásica de Beer (Monsi \& Saeki, 2005):

$$
f R F A I_{\text {_ }} \text { iaf }=1-\exp ^{-k i x i a l}
$$

donde ki adopta un valor constante de 0,57, de acuerdo a de la Casa et al. (2007).

La fracción de suelo cubierto por papa (f) se determinó a partir de fotografías tomadas de modo vertical sobre el cultivo y técnicas de interpretación de imágenes digitales (Rodríguez et al., 2000). El algoritmo de clasificación de las imágenes, que aplica el método de máxima verosimilitud (Eastman, 1997), prevé la identificación de cuatro clases: suelo y follaje, al sol y en penumbra, respectivamente. También se tomaron en este caso seis imágenes por tratamiento y fecha de muestreo. Cada fotografía vertical se tomó a una altura de 1,2 $\mathrm{m}$ en el mismo lugar de la lectura del interceptómetro, con el surco entre los camellones centrales como eje longitudinal medio. La estimación de fRFAl a partir de $f$ se realizó con la siguiente expresión obtenida por de la Casa et al. (2008b):

$$
f R F A I_{-} f=1-(1-f)^{0,7}
$$

donde 0,7 corresponde al cociente entre los coeficientes de extinción obtenidos con el interceptó- 
metro y la cobertura (ki/kf).

Los datos del ensayo se analizaron por correlación y regresión. La EUR se consideró igual a la pendiente lineal de la relación entre la materia seca total y la RFAl acumulada, y se obtuvo por medio de un análisis de regresión lineal asumiendo un valor nulo de intercepción.

\section{RESULTADOS Y DISCUSIÓN}

\section{Condiciones agrometeorológicas en el cinturón verde de Córdoba durante el ciclo 2008 de papa semitardía}

De acuerdo a la Figura 1, la radiación solar recibida por el cultivo de papa durante la temporada 2008 (ciclo semitardío) mostró las dos fluctuaciones características que se suscitan en esta época del año. Mientras a nivel diario se aprecia una variabilidad marcada de la energía recibida, de acuerdo a la dinámica propia de las condiciones de nubosidad y eventualmente lluvia, a largo plazo existe una paulatina disminución asociada con la marcha estacional de la radiación astronómica, como también muestra la Figura 1, para la región en esta época del año. De esta forma, si bien durante los primeros 50 días del ciclo el cultivo dispuso de mayor intensidad lumínica en términos potenciales, son más frecuentes las condiciones de tiempo nublado y eventualmente de lluvia, por lo que existen días de muy escasa recepción de radiación. Hacia la segunda parte del ciclo, y cuando el cultivo ya está establecido, la cantidad de radiación solar comienza a resultar naturalmente de menor intensidad.

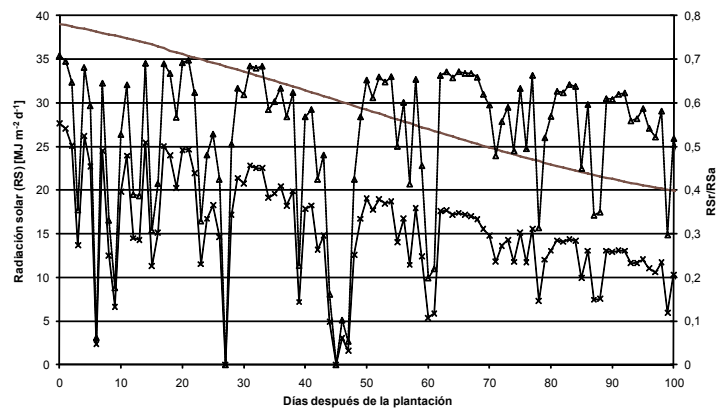

Figura 1: Radiación solar astronómica (RSa), recibida (RSr) y el cociente entre ambas (RSr/RSa) durante el ciclo del cultivo de papa a partir de la fecha de plantación (12/02/2008) en el cinturón verde de Córdoba, Argentina.

Como se observa en la Figura 2, las precipitaciones aportaron mayor cantidad de agua al cultivo durante los primeros 50 días del ciclo, totalizando

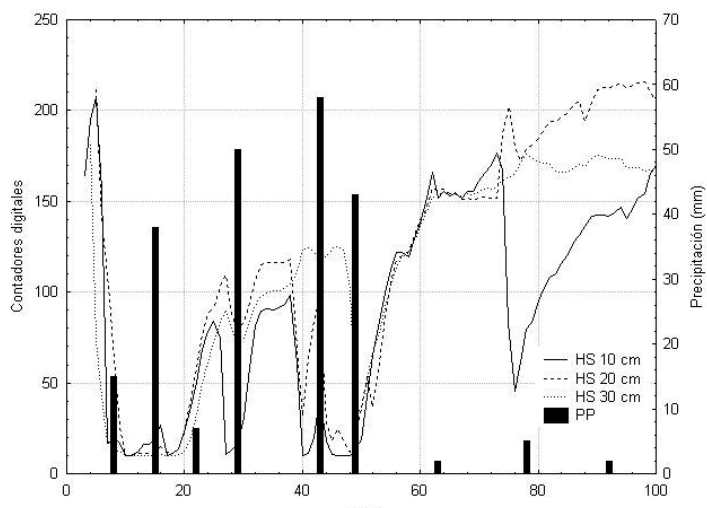

DDP

Figura 2: Variación de la humedad del suelo (HS) a 3 profundidades de acuerdo a la medición expresada en contadores digitales (CD) y la cantidad semanal de precipitación (PP) observados durante el ciclo del cultivo de papa a partir de la fecha de plantación (12/02/2008) en el cinturón verde de Córdoba, Argentina.

$211 \mathrm{~mm}$ en este periodo. Además, durante enero se registraron $233 \mathrm{~mm}$, lo que explica que el perfil estuviera húmedo a la plantación. Los sensores de humedad que se instalaron después de la plantación alcanzaron con cierto retraso un régimen de equilibrio con la humedad del suelo, de forma tal que el día 10 posterior a la plantación presenta la condición del perfil próximo a capacidad de campo (CC). Las mediciones de humedad de suelo están expresadas en contadores digitales, correspondiendo a CC un valor de 10 (el contenido de agua presenta una relación inversa con respecto a los contadores digitales). Durante la segunda mitad del ciclo, en cambio, las precipitaciones fueron muy escasas y ligeras.

El mayor aporte de la lluvia al comienzo del ciclo, tanto en términos de cantidad como de frecuencia, determinó que el contenido de agua del suelo fuera elevado durante este periodo, asegurando una adecuada provisión al cultivo. Si bien el contenido de agua no fue un elemento restrictivo para el crecimiento en esta parte del ciclo, durante la segunda mitad se produjo, en cambio, un paulatino decrecimiento de la humedad asociado al consumo del cultivo y la falta de reposición.

\section{Variación de la cobertura y el índice de área fo- liar durante el ciclo de papa para los distintos tratamientos}

Las curvas de la Figura 3 muestran la evolución de la parte aérea del cultivo de papa a lo largo del ciclo para los distintos tratamientos de densidad de plantación. Dichas curvas se presentan similares a las obtenidas por de la Casa et al. (2007) en 


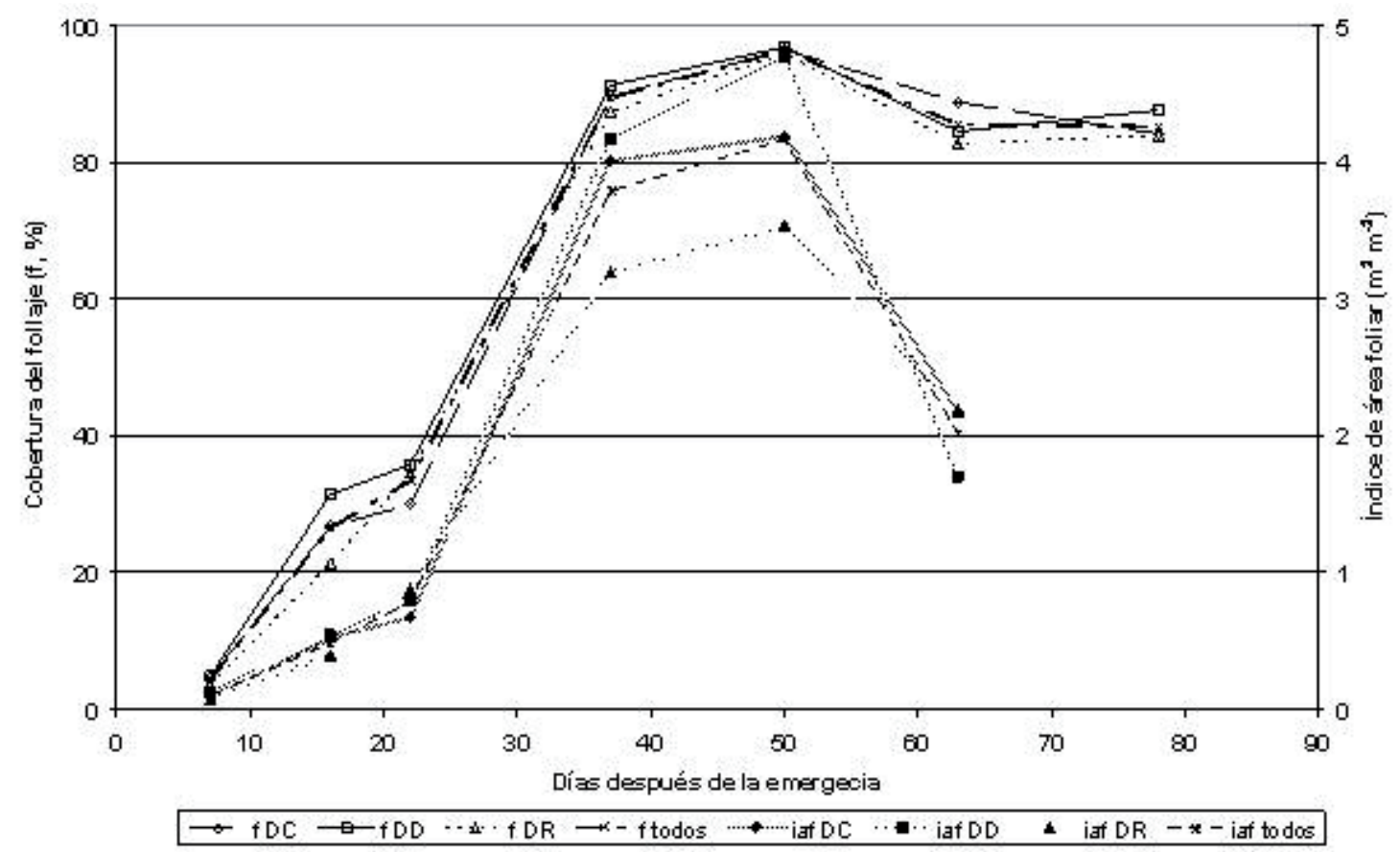

Figura 3: Variación de la cobertura (f) y del índice de área foliar (iaf) durante el ciclo 2008 de papa a partir de la emergencia (26/02/2008) en el cinturón verde de Córdoba, Argentina, para los distintos tratamientos de densidad de plantación (Doble (DD), comercial (DC) y raleada (DR)), y el promedio de los tratamientos (todos).

2005 bajo condiciones experimentales semejantes. Se observa que aproximadamente hasta la primera mitad del ciclo (día 50 después de la emergencia), tanto la cobertura como el iaf describen de manera equivalente el desarrollo vegetativo, manteniendo incluso el mismo ordenamiento de los respectivos tratamientos. Ambos parámetros son mayores en el caso de los cultivos bajo doble densidad (DD), menores en el tratamiento raleado (DR) e intermedios para los de densidad comercial (DC). Del mismo modo, las mediciones de $f y$ de iaf del tratamiento comercial resultan muy semejantes a los que se obtienen para un valor medio general de los tratamientos.

En el transcurso de la última parte del ciclo, se observa una caída más pronunciada del iaf en todos los tratamientos, en tanto $f$ mantiene valores superiores a $80 \%$ hasta el día 80 posterior a la emergencia. Esta diferencia se puede atribuir a la tendencia al vuelco que manifiesta el cultivo de papa cuando se encuentra completamente establecido, de forma tal que la fotografía capta material foliar sobre el suelo, en tanto pierde precisión la determinación del iaf que se realiza con la barra de intercepción. Estas dificultades para determinar los parámetros biofísicos en estudio de manera precisa, han sido reportados anteriormente por Haverkort et al. (1991) y Bouman et al. (1992).

No obstante, mientras la diferencia de los valores de cobertura entre los tratamientos se presenta

Tabla 1. Análisis de varianza para los valores de cobertura (f) y del índice de área foliar (iaf) en las distintas fechas de muestreo y tratamientos de densidad de plantación (DD: doble; DC: comercial; y DR: raleado), durante el ciclo del cultivo de papa a partir de la fecha de plantación (12/02/2008) en el cinturón verde de Córdoba, Argentina. F es el estadístico de Fisher con los grados de libertad del numerador y denominador, respectivamente; y p es el valor de probabilidad que la diferencia sea significativa.

\begin{tabular}{ccccccccccc}
\hline Fecha & & $f$ & & $F_{(2,15)}$ & $p$ & & iaf & & $F_{(2,15)}$ & $p$ \\
& DD & DC & DR & & & DD & DC & DR & & \\
\hline $04 / 03$ & 4,81 & 4,66 & 3,67 & 1,48 & 0,26 & 0,12 & 0,11 & 0,07 & 2,02 & 0,17 \\
$13 / 03$ & 31,38 & 26,71 & 21,35 & 3,26 & 0,07 & 0,54 & 0,52 & 0,40 & 1,91 & 0,18 \\
$19 / 03$ & 35,81 & 29,97 & 34,51 & 0,95 & 0,41 & 0,80 & 0,68 & 0,88 & 1,32 & 0,30 \\
$03 / 04$ & 91,31 & 89,66 & 87,33 & 1,29 & 0,30 & 4,17 & 4,01 & 3,19 & 1,35 & 0,29 \\
$16 / 04$ & 96,86 & 96,42 & 95,91 & 0,35 & 0,71 & 4,77 & 4,19 & 3,54 & 2,55 & 0,11 \\
$29 / 04$ & 84,52 & 88,79 & 82,68 & 1,35 & 0,29 & 1,70 & 2,18 & 2,18 & 2,10 & 0,16 \\
\hline
\end{tabular}




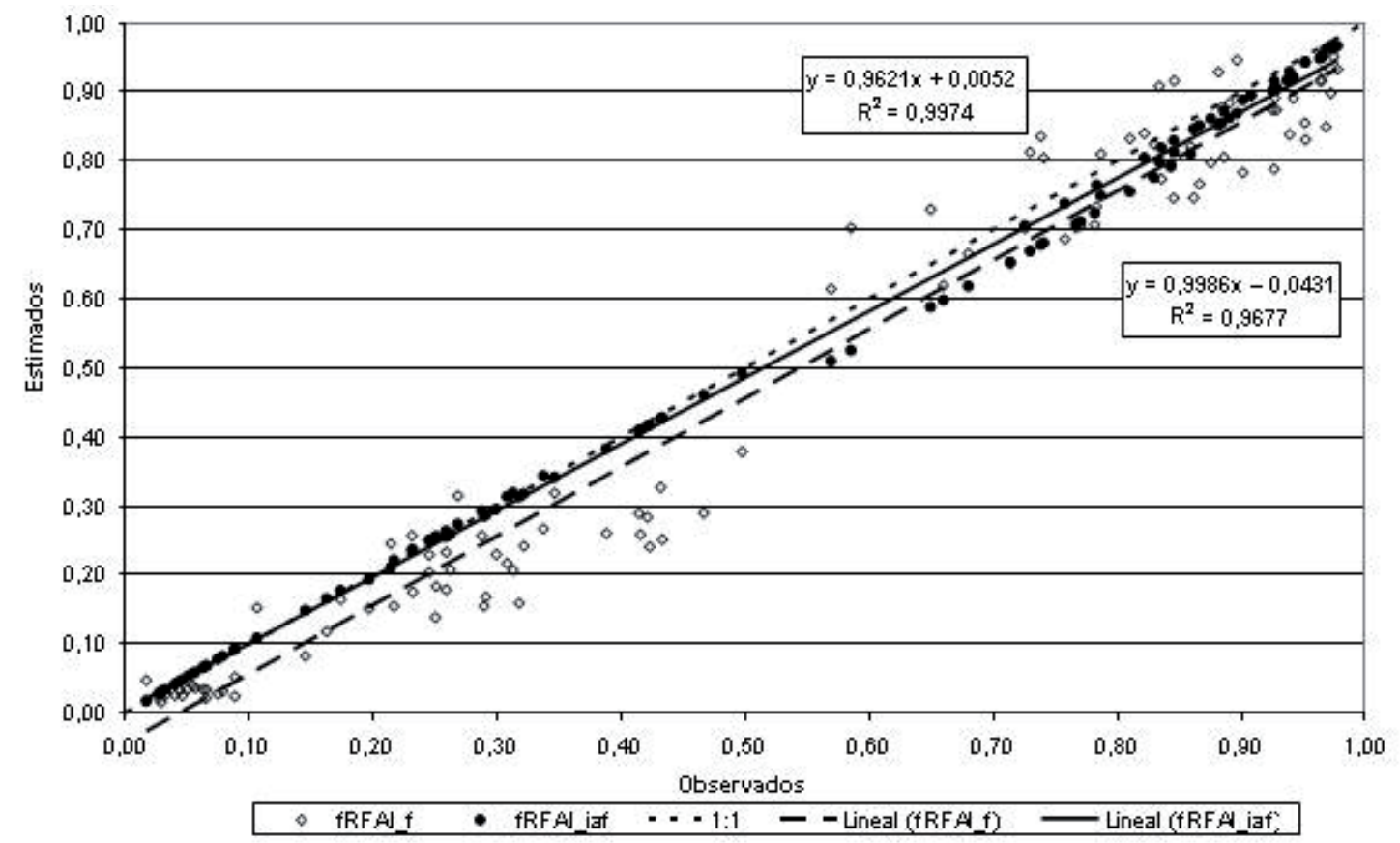

Figura 4: Relación entre los valores observados de la fracción de radiación fotosintéticamente activa interceptada por el cultivo de papa durante el ciclo 2008 en Córdoba, Argentina, y los estimados a partir de la cobertura del follaje (fRFAl_f) y del índice de área foliar (fRFAl_iaf) para el conjunto completo de mediciones. También se incluye la recta de identidad (1:1).

menos notable y para ninguna fecha resulta significativa $(P<0,05)$, como indica la Tabla 1, los del iaf muestran un rango más pronunciado, aunque sólo cuando el cultivo alcanza valores superiores a 3. Se debe tener en cuenta que, dada su relación exponencial con la fRFAl, los valores de iaf superiores a 3 producen sólo un ligero aumento de la intercepción. Sin embargo, a pesar de la mayor diferencia aparente entre los tratamientos, tampoco los valores de iaf acusaron diferencias significativas $(P<0,05)$ (Tabla 1), y presentaron una dispersión considerable en el muestreo (datos no mostrados). De acuerdo a estos resultados, la determinación de la EUR se realizó a partir de un promedio general de todas las mediciones.

\section{EUR determinada con valores medidos de fRFAI y calculada a partir de iaf $\mathbf{y} \mathbf{f}$}

La propuesta de utilizar los valores de cobertura del follaje $(f)$, que es una medición relativamente más fácil y económica de realizar que la del iaf, a los efectos de determinar la fracción interceptada de radiación fotosintéticamente activa, requiere contrastar los resultados obtenidos a partir de ambos parámetros biofísicos. La Figura 4 muestra la relación entre los valores observados de la fracción de radiación fotosintéticamente activa que fueron medidos en todos los tratamientos durante el ciclo completo, respecto a los valores estimados tanto a partir de $f\left(f R F A I \_f\right)$ como de iaf (fRFAl_iaf). El análisis de correlación y regresión, cuyos resultados también muestra la Figura 4, sintetiza las principales diferencias entre ambos procedimientos.

La menor dispersión de los valores obtenidos con el método que utiliza el iaf responde a lo esperado, en razón de que las determinaciones de iaf fueron obtenidas con el medidor del flujo de fotones, es decir bajo el mismo principio fundado en la ley de Beer y a partir de los mismos datos de intercepción. Mientras la ordenada al origen es prácticamente nula, la recta de regresión presenta un valor ligeramente menor a 1. En contraposición, existe mayor dispersión en los datos cuando se utiliza la cobertura como estimador de fRFAl, si bien el $R^{2}$ presenta un valor superior a 0,96 $(P<0,05)$, lo que representa un ajuste muy consistente. Aunque el coeficiente angular es prácticamente igual a 1, la recta de regresión presenta un valor de ordenada que difiere aproximadamente $5 \%$ del origen, lo que conduce a la subestimación de la radiación interceptada al comienzo del ciclo, cuando la recepción es más importante (Figura 1) y, en consecuencia, traslada ese error al cálculo de la EUR.

La Figura 5 presenta la resolución gráfica de los valores de EUR obtenidos de acuerdo al análisis de regresión lineal entre lo valores acumulados de 


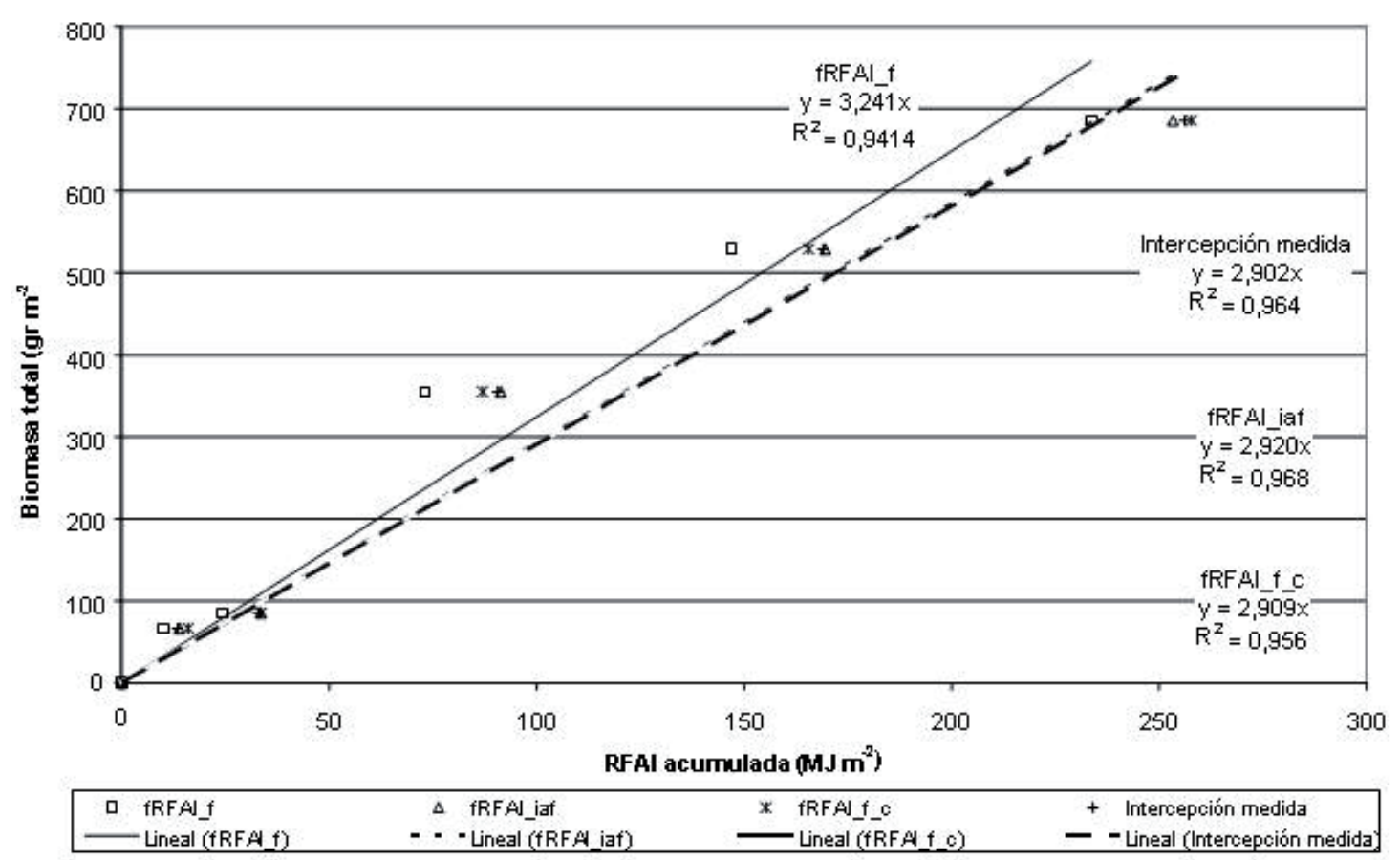

Figura 5: Relación entre la radiación fotosintéticamente activa interceptada (RFAI) y la biomasa total del cultivo de papa durante el ciclo 2008 en Córdoba, Argentina. Todas las pendientes son significativas $(P<0,01)$.

radiación fotosintéticamente activa interceptada que se obtuvieron a partir de los distintos métodos de medición o estimación y la biomasa total producida (parte aérea y subterránea). Considerando sólo el espectro fotosintéticamente activo, la EUR obtenida como la pendiente lineal a partir de los valores medidos de la fracción de radiación interceptada fue de 2,90 $\mathrm{gr} \mathrm{MJ}^{-1}$ PAR (1,45 $\mathrm{gr} \mathrm{MJ}^{-1}$ solar) $(P<0,01)$, valor que se asume de referencia. Este valor de EUR es similar al que Kooman et al. (1996) determinaron en Tunes durante el ciclo otoñal de producción de papa, es decir bajo condiciones climáticas semejantes a las de este ensayo.

La EUR determinada a partir de la fRFAl_iaf produce una pendiente exactamente similar a la que se obtiene utilizando los valores medidos de intercepción, lo que se justifica por la razón ya señalada. En cambio, el valor de EUR que se obtiene usando la fRFAl_f produce un valor de 3,2 gr $\mathrm{MJ}^{-1}$, que está por encima del valor de referencia para esta experiencia. La diferencia se explica, de acuerdo al análisis previo (Figura 4), en razón de la subestimación sistemática de la fracción de intercepción que origina este método, principalmente hacia el comienzo del ciclo. De esta forma, la cantidad de radiación acumulada es más reducida, lo que aumenta de manera errónea la tasa potencial de crecimiento. Si la fracción de radiación interceptada se incrementa el 5\%, de acuerdo al valor de ordenada que se obtuvo del análisis en la Figura 4, la línea de regresión se superpone a la del método de referencia, como se indica en la Figura 5, y las tasas de eficiencia estimadas resultan idénticas.

De acuerdo a los valores de EUR obtenidos para el cultivo de papa en distintas experiencias, la tasa de 1,45 $\mathrm{g} \mathrm{MJ}^{-1}$ solar corresponde al valor inferior de un rango que se extiende entre 1,45 y $1,7 \mathrm{~g} \mathrm{MJ}^{-1}$ solar (Sinclair \& Muchow, 1999), aunque Kooman et al. (1996) reportan valores de EUR aún más bajos y también superiores. De todos modos, la tasa relativamente baja obtenida en este caso refleja la condición de estrés hídrico que experimentó el cultivo al final del ciclo, como se deduce de la Figura 2.

Asimismo, la variación de la biomasa acusa un comportamiento no estrictamente lineal respecto a la radiación acumulada, lo que también es un indicio en este sentido, de manera similar a lo que Jefferies \& Mackerron (1989) observan en sus tratamientos con sequía impuesta. Siguiendo ese razonamiento, a partir del contenido de agua para un perfil de $50 \mathrm{~cm}$ de profundidad, obtenido de las mediciones de potencial y la curva de calibración correspondiente al suelo de la experiencia, y adop- 


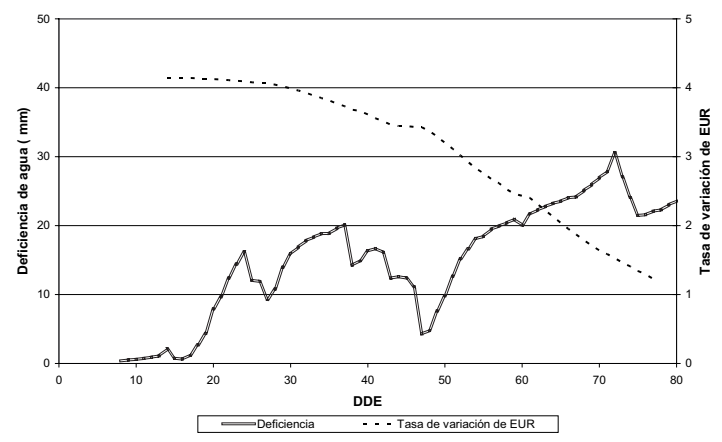

Figura 6: Deficiencia de agua en el perfil y tasa de variación de la EUR respecto a los días después de la emergencia (DDE), durante el ciclo 2008 de papa en Córdoba, Argentina.

tando un valor de capacidad de campo de $28 \%$, se determinó la deficiencia de agua de acuerdo a CC-HS. Por su parte, la tasa de cambio de la EUR para cada fecha se calculó a partir de la derivada de la curva de ajuste (para un polinomio de $2^{\circ}$ grado con ordenada nula) entre la biomasa y la radiación interceptada. La representación conjunta de ambas variables durante los 80 días posteriores a la emergencia, como muestra la Figura 6 , indica un valor siempre decreciente de la tasa de cambio de EUR respecto a la radiación interceptada. Aproximadamente después de 60 días posteriores a la emergencia y con una deficiencia de agua superior a $20 \mathrm{~mm}$, los valores diarios de EUR resultan claramente disminuidos por debajo de la tasa máxima de 3,5 $\mathrm{g} \mathrm{MJ}^{-1}$ (RFA) (1,75 $\mathrm{g} \mathrm{MJ}^{-1}$ solar) (Griffin et al., 1993) en respuesta al estrés hídrico.

Estos resultados son consistentes porque no sólo verifican la metodología de análisis utilizada por Jefferies \& Mackerron (1989), sino que también confirman que un potencial matriz inferior a -70 cbar constituye el umbral de estrés hídrico en papa para las condiciones experimentales del cinturón verde de Córdoba, de acuerdo a resultados previos bajo condiciones experimentales similares (de la Casa et al., 2001).

\section{CONCLUSIONES}

La utilización de la cobertura del follaje para representar la fracción de radiación interceptada en papa produjo resultados que sobreestimaron el valor de EUR, principalmente como consecuencia de una sistemática subestimación de la fracción de radiación interceptada. Si bien la utilización de la cobertura puede ser un recurso válido para evaluar la dinámica del crecimiento bajo distintos tratamientos, y su empleo no requiere de instrumental específico ni depende de las condiciones de ilu- minación, lo que le confiere un carácter particularmente ventajoso en términos operativos, los valores de $f R F A l \_f$ debieron ser corregidos para producir resultados de EUR similares al de referencia.

\section{BIBLIOGRAFÍA}

AccuPAR, Opertor's Manual Version 3.4, 2001. Linear PAR/LAl ceptometer. Model PAR- 80.Decagon Devices, Inc. Pullman, WA, USA.

Bouman, B.A.M., D. Uenk and A.J. Haverkort. 1992. The estimation of ground cover of potato by reflectance measurements. Potato Res. 35:111-125.

Brisson, N., C. Gary, E. Justes, R. Roche, B. Mary, D. Ripoche, D. Zimmer, J. Sierra, P. Bertuzzi, P. Burger, F. Bussière Y.M. Cabidoche, P. Cellier, P. Debaeke, J.P. Gaudille're, C. He'nault, F. Maraux, B. Seguin and H. Sinoquet. 2003. An overview of the crop model STICS. Europ. J. Agronomy 18:309-332.

Caviglia, O.P., V.O. Sadras and F.H. Andrade. 2003. Intensification of agriculture in the south-eastern Pampas: I. Capture and efficiency in the use of water and radiation in double-cropped wheat-soybean. Field Crops Res. 87:117-129.

Dardanelli, J.D., O.A. Bachmeier, R. Sereno and R. Gil. 1997. Rooting depth and soil water extraction patterns of different crops in a silty loam Haplustoll. Field Crop Res. 54:29-38.

de la Casa, A., A. Rodríguez, L. Bressanini, R. Accietto y G. Ovando, 2001. Efecto del estrés hídrico en papa (Solanum tuberosum L.) sobre el índice de área foliar. Revista Argentina de Agrometeorología 1(2):43-47.

de la Casa, A., G. Ovando, L. Bressanini, Á. Rodríguez y J. Martínez. 2007. Uso del índice de área foliar y del porcentaje de cobertura del suelo para estimar la radiación interceptada en papa. Agricultura Técnica (Chile) 67(1):78-85.

de la Casa, A., G. Ovando, L. Bressanini, J. Martínez, E. Ibarra y Á. Rodríguez. 2008a. El índice de área foliar en papa estimado a partir de la cobertura del follaje. Agronomía Tropical 58(1):61-64.

de la Casa, A., G. Ovando, L. Bressanini, J. Martínez y Á. Rodríguez. 2008b. Evaluación de un modelo para estimar la fracción de radiación interceptada en papa (Solanum tuberosum L.) bajo distintas densidades de plantación. XIII Reunión Argentina de Agrometeorología. 8 al 10 de octubre de 2008. San Salvador de Jujuy, Argentina.

Demetriades-Shaw, T.H., M. Fuchs, E.T. Kanemasu and I. Flitcroft. 1992. A note of caution concerning the relationship between cumulated intercepted solar radiation and crop growth. Agric. For. Meteorol. 58:193-207.

Demetriades-Shaw, T.H., M. Fuchs, E.T. Kanemasu and I. Flitcroft. 1994. Further discussions on the relationship between cumulated intercepted solar radiation and crop growth. Agric. For. Meteorol. 68:231-242. 
Duffie, J.A. and W.A. Beckman. 1980. Solar engineering of thermal processes. John Wiley and Sons, New York. pp. 1-109.

Eastman, R.J. 1997. Clasification of remotely sensed imagery. p. 1-29. In.IDRISI: User's guide Idrisi for Windows. Clark University, Worcester, Massachusetts, USA.

Edwards, J.T., L.C. Purcell and E.D. Vories. 2005. Light interception and yield potential of short-season maize (Zea mays L.) hybrids in the midsouth. Agron. J. 97:225-234.

Gallo, K.P., C.S.T. Daughtry and C.L. Wiegand. 1993. Errors in measuring absorbed radiation and computing crop radiation use efficiency. Agron. J. 85:12221228.

Griffin, T.S., B.S. Johnson and J.T. Ritchie. 1993. A simulation model for potato growth and development: Substor-potato Version 2.0. IBSNAT Research Report Series 02. Dept. of Agronomy and Soil Science.

Haverkort, A.J., D. Uenk, H. Veroude and M. Van de Waart. 1991. Relationships between ground cover, intercepted solar radiation, leaf area index and infrared reflectance of potato crops. Potato Res. 34:113-121.

Jarsún, B., J. Gorgas, E. Zamora, H. Bosnero, E. Lovera, A. Ravelo y J. Tassile. 2006. Los suelos de Córdoba. Agencia Córdoba Ambiente e Instituto Nacional de Tecnología Agropecuaria, EEA Manfredi. Córdoba, Argentina.

Jefferies, R.A. and D.K.L. Mackerron. 1989. Radiation interception and growth of irrigated and droghted potato (Solanum tuberosum L.). Field Crops Res. 22:101-112.

Jefferies, R.A. and T.D. Heilbronn. 1991. Water stress as a constraint on growth in the potato crop. 1 Model development. Agric. For. Meteorol. 53:185-196.

Jonckheere, I., S. Fleck, K. Nackaerts, B. Muys, P. Coppin, M. Weiss and F. Baret. 2004. Review of methods for in situ leaf area index determination. Part I. Theories, sensors and hemispherical photography. Agric. For. Meteorol. 121:19-35.

Kadaja, J. and H. Tooming. 2004. Potato production model based on principle of maximum plant productivity. Agric. For. Meteorol. 127:17-33.

Kooman, P.L., M. Fahem, P. Tegera and A.J. Haverkort. 1996. Effects of climate on different potato genotypes.1. Radiation interception, total and tuber dry matter production. Europ. J. Agronomy 5:193-205.
Lindquist, J.L., T.J. Arkebauer, D.T. Walters, K.G. Cassman and A. Dobermann. 2005. Maize radiation use efficiency under optimal growth conditions. Agron. J. 97:72-78.

Liu, J., J.R. Miller, E. Pattey, D. Haboudane, I.B. Strachan and M. Hinther. 2004. Monitoring crop biomass accumulation using multi-temporal hyperspectral remote sensing data. IEEE, 1637-1640.

Loomis, R.S. and J.S. Amthor. 1999. Yield potential, plant assimilatory capacity, and metabolic efficiencies. Crop Sci. 39:1584-1596.

Monsi, M. and T. Saeki. 2005. On the factor light in plant communities and its importance for matter production. Annals of Botany 95: 549-567.

Monteith, J.L. 1977. Climate and the efficiency of crop production. Britain. Phil. Trans. R. Soc. Lond. p. 277294.

Monteith, J.L. 1994. Validity of the correlation between intercepted radiation and biomass. Agric. For. Meteorol. 68:213-220.

Purcell, L.C., 2000. Soybean canopy coverage and light interception measurements using digital imagery. Crop Science 40:834-837.

Rodríguez, A., A. de la Casa, R. Accietto, G. Ovando y L. Bressanini.1997. La oportunidad del riego determinada por sensores de matriz granular (smg) en relación a los cambios de la condición de cobertura del cultivo de papa (Solanum tuberosum, cv Spunta) afectado por sequía. I Reunión Latinoamericana y VII Reunión Argentina de Agrometeorología. Facultad de Agronomía (UBA) y Asociación Argentina de Agrometeorología. 28-30 de abril de 1997. Buenos Aires, Argentina.

Rodríguez, A., A. de la Casa, R. Accietto, L. Bressanini y G. Ovando. 2000. Determinación del área foliar en papa (Solanum tuberosum L., var.Spunta) por medio de fotografías digitales conociendo la relación entre el número de pixeles y la altura de adquisición. Revista Brasileira de Agrometeorología 8:215-221.

Sinclair, T.R. and R.C. Muchow. 1999. Radiation use efficiency. Adv. in Agron. 65:215-265.

Steven, M.D., P.V. Biscoe, K.W. Jaggard and J. Paruntu. 1986. Foliage cover and radiation interception. Field Crop Res. 13:75-87.

Van Oijen, M. 1991. Light use efficiencies of potato cultivars with late blight (Phytophthora infestans). Potato Res. 34:123-132. 